\title{
Using a New Entropy Loss Analysis to Assess the Accuracy of RANS Predictions of an HPT Vane
}

\author{
Yaomin Zhao* \\ Department of Mechanical Engineering \\ University of Melbourne \\ Melbourne, VIC 3010, Australia \\ Email: yaomin.zhao@unimelb.edu.au \\ Richard D. Sandberg \\ Department of Mechanical Engineering \\ University of Melbourne \\ Melbourne, VIC 3010, Australia \\ Email: richard.sandberg@unimelb.edu.au
}

\begin{abstract}
Entropy loss is widely used to quantify the efficiency of components in turbomachines, and empirical relations have been developed to estimate the contribution of different mechanisms. However, further analysis is still needed to not only get a deeper insight of the physics, but also to more accurately quantify the loss generation caused by different terms. In the present study, the entropy transport equations based on averaged flow quantities are first derived, and the entropy generation process is fully decomposed into several terms representing different physical mechanisms, such as mean viscous dissipation, turbulence production, mean and turbulent heat flux, etc. This decomposition framework is then applied to high-resolution LES and RANS results of a VKI LS-89 HPT vane, and a detailed quantification of different entropy generation terms is obtained. The results show that the entropy generation caused by mean flow features like mean viscous dissipation and mean heat flux are in close agreement between LES and RANS, indicating that RANS provides an overall good prediction for the mean flow. Furthermore, we find that turbulence production plays an important role in entropy generation as it represents the energy extracted from the mean flow to
\end{abstract}

*Address all correspondence to this author. 
turbulent fluctuations. However, the difference between RANS and LES results for the turbulence production term is not negligible, particularly in the wake region. This implies that the failure of RANS to predict the correct total loss might be largely caused by errors in capturing the correct turbulence production in the near wake region.

\section{INTRODUCTION}

An efficient use of energy is one of the most important goals for designing modern turbomachines. Based on the second law of thermodynamics, entropy generation is widely used to represent loss of efficiency [1,2]. Generally, viscous effects and irreversible heat flux are taken as major sources for entropy generation in turbomachines, and various empirical relations have been derived to estimate the entropy generated from different origins such as the viscous effects in the boundary layer, viscous effects in wake mixing, and heat transfer across temperature differences [3]. However, the accuracy of these correlation based methods is limited in many cases. Therefore, further analysis is still needed not only to get a deeper insight of the physics, but also to more accurately quantify the loss generation caused by different terms.

Thanks to the fast development of numerical algorithms, turbulence modelling techniques, and computational power in the past decades, computational fluid dynamics (CFD), despite some limitations, is playing a more and more important role in turbomachine designs [4]. Calculating entropy generation based on CFD flow fields can provide not only the total entropy variation, but also the spatial distribution of local entropy generation. Therefore, CFD analysis promises to be a valuable tool for the prediction of efficiency in turbomachinery components, which is very useful for the design community $[5,6]$.

In previous studies, different methods have been proposed to quantify entropy generation mechanisms based on CFD calculations. Moore and Moore [7] first proposed a method to calculate mean entropy generation with viscous dissipation and increase of turbulent kinetic energy (TKE), and the method was then applied, showing satisfactory results for entropy generation in a two-dimensional turbulent boundary layer. Camberos [8] presented a strategy to derive entropy balance equations for general thermodynamic processes based on the second law, and entropy generation in instantaneous flow fields was presented. Reynolds averaging techniques were applied to the instantaneous entropy generation equations, and the modelling of turbulence closures in entropy transport equations for viscous, incompressible flows was proposed [9]. More recently, the entropy generation in a high-pressure turbine (HPT) vane was obtained by post-processing direct numerical simulation (DNS) data, and the individual contribution from the different sources were quantified [10]. Lengani et al. [11] derived an entropy balance equation expressed as a 
function of total quantities, and a proper orthogonal decomposition procedure was applied to a large-eddy simulation (LES) of a low-pressure turbine (LPT) to determine the dominating modes for the entropy generation. It is noted that the entropy breakdown closures derived from previous studies have been presented in various forms. The lack of consistency, however, limits the applicability of the entropy analysis.

Here, we present a complete derivation of the entropy balance equation for compressible flows in turbomachines. The closure is derived from basic flow governing equations and thermodynamic laws, which guarantees the framework can be applied to both high-fidelity data and RANS modelling results. Applying this entropy analysis to a LS-89 HPT vane, the accuracy of RANS predictions will be assessed based on high-resolution LES data. The error of RANS modelling for turbulence closures are quantified based on entropy generation, and the major error source within the vane is located.

\section{ENTROPY BALANCE EQUATIONS}

Based on the second law of thermodynamics, the total entropy $S$ of a system satisfies

$$
\dot{S}+I_{S}=\int_{V} G_{s} d V \geq 0
$$

where $\dot{S}$ is the time-rate-of-change of the system entropy, $I_{S}$ is the entropy current with external environment, and the right-hand side term represents the entropy generation rate $G_{s}$ integrated over the volume $V$ [8]. For most thermodynamic processes, the generation of total entropy is greater than zero, while the equivalent holds only when the process is reversible.

For single phase fluid flows, the entropy balance equation can be written as

$$
\frac{\partial(\rho s)}{\partial t}+\frac{\partial F_{i}}{\partial x_{i}} \equiv G_{s} \geq 0
$$

Here, $\rho$ is density of the local fluid, $s$ is the specific entropy, and $F_{i}$ is the entropy flux caused by mass flux and heat transfer [9]. It is noted that the specific form of $F_{i}$ and $G_{s}$ depends on the governing equations for the flow. For example, the entropy generation mechanisms for viscous or inviscid flows will be significantly different.

In the following, we will present the derivation of entropy generation equations for turbine flows. 


\section{Entropy Generation with Navier-Stokes Equations}

This part presents the process to derive the local entropy balance equation based on the three dimensional compressible Navier-Stokes (NS) equations. First of all, the conservation of mass, momentum and energy can be written as

$$
\begin{gathered}
\frac{\partial \rho}{\partial t}+\frac{\partial\left(\rho u_{j}\right)}{\partial x_{j}}=0 \\
\frac{\partial\left(\rho u_{i}\right)}{\partial t}+\frac{\partial\left(\rho u_{i} u_{j}+p \delta_{i j}\right)}{\partial x_{j}}=\frac{\partial \sigma_{i j}}{\partial x_{j}}, \\
\frac{\partial\left(\rho e_{0}\right)}{\partial t}+\frac{\partial\left[u_{j}\left(\rho e_{0}+p\right)\right]}{\partial x_{j}}=\frac{\partial\left(\sigma_{i j} u_{i}\right)}{\partial x_{j}}-\frac{\partial q_{j}}{\partial x_{j}},
\end{gathered}
$$

with $u_{i}, p, e_{0}$ representing velocity components, pressure, and specific energy, respectively. Here, the fluid is assumed to be Newtonian with second viscosity set to $-2 / 3 \mu$, so that the viscous stress is written as

$$
\sigma_{i j}=\mu\left(-\frac{2}{3} \frac{\partial u_{k}}{\partial x_{k}} \delta_{i j}+\frac{\partial u_{i}}{\partial x_{j}}+\frac{\partial u_{j}}{\partial x_{i}}\right)
$$

Moreover, using the Fourier law, the heat flux vector components are given by

$$
q_{j}=-\frac{c_{p} \mu}{\operatorname{Pr}} \frac{\partial T}{\partial x_{j}}
$$

where $c_{p}$ is specific heat at constant pressure, and $\operatorname{Pr}$ is the Prandtl number.

Subtracting the kinetic energy from the total energy equation, i. e. $(5)-u_{i} \times(4)+\frac{1}{2} u_{i} u_{i} \times(3)$, the 
equation for the internal energy $e=e_{0}-1 / 2 u_{i} u_{i}$ can be derived as

$$
\frac{\partial(\rho e)}{\partial t}+\frac{\partial\left(\rho u_{j} e\right)}{\partial x_{j}}+p \frac{\partial u_{j}}{\partial x_{j}}=\sigma_{i j} \frac{\partial u_{i}}{\partial x_{j}}-\frac{\partial q_{j}}{\partial x_{j}}
$$

Combining the first law of thermodynamics

$$
d e=T d s-p d \frac{1}{\rho}
$$

with Eqn. (8), the entropy balance equation can be derived

$$
\begin{aligned}
\frac{\partial(\rho s)}{\partial t} & +\frac{\partial\left(\rho u_{i} s\right)}{\partial x_{i}}+\frac{\partial}{\partial x_{i}}\left(\frac{q_{i}}{T}\right) \\
& =\frac{1}{T} \sigma_{i j} \frac{\partial u_{j}}{\partial x_{i}}+\frac{c_{p} \mu}{\operatorname{Pr}} \frac{1}{T^{2}}\left(\frac{\partial T}{\partial x_{i}}\right)^{2} .
\end{aligned}
$$

Compared to the generalized entropy balance equation Eqn. (2), the entropy flux for compressible viscous flow is caused by mass flux and heat flux as

$$
\frac{\partial F_{i}}{\partial x_{i}}=\underbrace{\frac{\partial\left(\rho u_{i} s\right)}{\partial x_{i}}}_{\text {mass flux }}+\underbrace{\frac{\partial}{\partial x_{i}}\left(\frac{q_{i}}{T}\right)}_{\text {heat flux }},
$$

and the entropy generation mechanisms are related to viscous dissipation and irreversible heat transfer

$$
G_{s}^{N S}=\underbrace{\frac{1}{T} \sigma_{i j} \frac{\partial u_{j}}{\partial x_{i}}}_{\text {vis. dissip. }}+\underbrace{\frac{c_{p} \mu}{P r} \frac{1}{T^{2}}\left(\frac{\partial T}{\partial x_{i}}\right)^{2}}_{\text {heat trans. }} .
$$

We note that the above term $G_{s}^{N S}$ applies to the local entropy generation in the instantaneous flow fields governed by Navier-Stokes equations. Taking the average of the instantaneous entropy generation Eqn. (12), we can decompose the viscous effect into mean flow viscous dissipation and turbulent dissipa- 
tion [10]. However, other terms related to turbulent fluctuations, such as the turbulent heat flux, cannot be directly derived. Furthermore, due to the extremely complex environment in turbomachines, flows experience highly unsteady, non-equilibrium thermodynamic processes through turbine vanes, particularly with strong turbulence and non-uniform distribution of the fluid. Therefore, a volume integral of the local entropy generation term in Eqn. (12) might be biased for the total entropy generation of the whole system.

\section{Entropy Loss with Favre-Averaged NS Equations}

The efficiency of turbomachines is generally assessed in a time-averaged sense. Therefore, the entropy generation should be calculated based on mean flow fields, $i$. e. RANS results or averaged fields from highfidelity data. Accordingly, the Favre-averaged conservation equations [12] need to be considered.

$$
\begin{gathered}
\frac{\partial \bar{\rho}}{\partial t}+\frac{\partial\left(\bar{\rho} \widetilde{u}_{j}\right)}{\partial x_{j}}=0 \\
\frac{\partial\left(\bar{\rho} \widetilde{u}_{i}\right)}{\partial t}+\frac{\partial\left(\bar{\rho} \widetilde{u}_{i} \widetilde{u}_{j}+\bar{p} \delta_{i j}\right)}{\partial x_{j}}=\frac{\partial \sigma_{i j}(\widetilde{u})}{\partial x_{j}}+\frac{\partial \tau_{i j}^{R}}{\partial x_{j}}, \\
\frac{\partial}{\partial t}\left(\bar{\rho} \widetilde{e}+\frac{1}{2} \bar{\rho} \widetilde{u}_{i} \widetilde{u}_{i}+\bar{\rho} k\right)+\frac{\partial}{\partial x_{j}}\left[\widetilde{u}_{j}\left(\bar{\rho} \widetilde{e}+\frac{1}{2} \bar{\rho} \widetilde{u}_{i} \widetilde{u}_{i}+\bar{\rho} \bar{k}+\bar{p}\right)\right] \\
=\frac{\partial}{\partial x_{j}}\left(\widetilde{u}_{i} \sigma_{i j}(\widetilde{u})+\widetilde{u}_{i} \tau_{i j}^{R}\right)-\frac{\partial}{\partial x_{j}}\left(\widetilde{q}_{j}+q_{j}^{R}\right) \\
+\frac{\partial \mathcal{F}_{j}}{\partial x_{j}}+\frac{\partial}{\partial x_{j}}[\mathcal{V}] .
\end{gathered}
$$

Here, $\overline{\mathcal{C}}$ is the time-average of $\mathcal{C}$, and $\widetilde{\mathcal{C}}$ denotes a Favre-averaged quantity as

$$
\widetilde{\mathcal{C}}=\frac{\overline{\rho \mathcal{C}}}{\bar{\rho}}
$$


Journal of Turbomachinery

The Reynolds stress is

$$
\tau_{i j}^{R}=-\bar{\rho}\left(\widetilde{u_{i} u_{j}}-\widetilde{u}_{i} \widetilde{u}_{j}\right)
$$

and the turbulent heat flux is

$$
q_{j}^{R}=\bar{\rho} c_{p}\left(\widetilde{T u_{j}}-\widetilde{T} \widetilde{u}_{j}\right)
$$

These two terms represent the contribution of fluctuations to the mean flow, which are typically modelled in RANS calculations. Furthermore, the term $\mathcal{V}$ is the viscous effect related to fluctuation velocities as

$$
\mathcal{V}=\overline{u_{i}^{\prime \prime}} \sigma_{i j}(\widetilde{u})+\widetilde{u}_{i} \sigma_{i j}\left(\overline{u^{\prime \prime}}\right)
$$

which is negligible for most cases. Moreover, the term $\mathcal{F}_{j}$ is the total flux for the TKE $k=0.5 \overline{\rho u_{i}^{\prime \prime} u_{i}^{\prime \prime}} / \bar{\rho}$, which can be decomposed as

$$
\mathcal{F}_{j}=-\overline{\rho u_{j}^{\prime \prime} \frac{1}{2} u_{i}^{\prime \prime} u_{i}^{\prime \prime}}-\overline{p^{\prime} u_{i}^{\prime \prime}} \delta_{i j}+\overline{u_{i}^{\prime \prime} \sigma_{i j}\left(u^{\prime \prime}\right)}
$$

Here, $u_{i}^{\prime \prime}=u_{i}-\widetilde{u}_{i}$, and $p^{\prime}=p-\bar{p}$. Note that the volume integral of $\int_{V} \frac{\partial \mathcal{F}_{j}}{\partial x_{j}} d V$ is usually negligible in turbine flows, so that the TKE flux terms can be neglected for the entropy calculations in the present study.

For time-averaged flow fields, the time derivative terms in Eqns. (13), (14) and (15) equal to zero, and we can assume that the mean flow governed by the equations satisfies the first thermodynamic law as

$$
d \widetilde{e}=\widetilde{T} d \widetilde{s}-\bar{p} d \frac{1}{\bar{\rho}}
$$

Furthermore, discarding the negligible terms with $\mathcal{V}$ and $\mathcal{F}_{j}$ in Eqn. (15), we can derive the entropy balance 
equation for the mean flow via a similar process for the instantaneous equation

$$
\begin{aligned}
\frac{\partial\left(\bar{\rho} \widetilde{u}_{i} \widetilde{s}\right)}{\partial x_{i}}+ & \frac{\partial}{\partial x_{i}}\left(\frac{\widetilde{q}_{i}}{\widetilde{T}}\right)=\frac{1}{\widetilde{T}} \sigma_{i j}(\widetilde{u}) \frac{\partial \widetilde{u}_{j}}{\partial x_{i}}+\frac{c_{p} \mu}{\operatorname{Pr}} \frac{1}{\widetilde{T}^{2}}\left(\frac{\partial \widetilde{T}}{\partial x_{i}}\right)^{2} \\
& +\frac{1}{\widetilde{T}} \tau_{i j}^{R} \frac{\partial \widetilde{u}_{j}}{\partial x_{i}}-\frac{1}{\widetilde{T}} \frac{\partial}{\partial x_{i}}\left(q_{i}^{R}\right)-\frac{1}{\widetilde{T}} \frac{\partial\left(\bar{\rho} \widetilde{u}_{i} k\right)}{\partial x_{i}} .
\end{aligned}
$$

Similar to Eqn. (11), the left-hand side terms represent entropy variations caused by mass and heat fluxes with external environments. On the right-hand side, the entropy generation mechanisms for RANS flow fields can be written as

$$
\begin{aligned}
& \widetilde{G}_{s}^{R A N S}=\underbrace{\frac{1}{\widetilde{T}} \sigma_{i j}(\widetilde{u}) \frac{\partial \widetilde{u}_{j}}{\partial x_{i}}}_{V_{M}}+\underbrace{\frac{c_{p} \mu}{\operatorname{Pr}} \frac{1}{\widetilde{T}^{2}}\left(\frac{\partial \widetilde{T}}{\partial x_{i}}\right)^{2}}_{H_{M}} \\
& +\underbrace{\frac{1}{\widetilde{T}} \tau_{i j}^{R} \frac{\partial \widetilde{u}_{j}}{\partial x_{i}}}_{P_{R}}-\underbrace{\frac{1}{\widetilde{T}} \frac{\partial}{\partial x_{i}}\left(q_{i}^{R}\right)}_{H_{R}}-\underbrace{\frac{1}{\widetilde{T}} \frac{\partial\left(\bar{\rho} \widetilde{u}_{i} k\right)}{\partial x_{i}}}_{A_{k}}
\end{aligned}
$$

which include entropy generation caused by the mean viscous dissipation $V_{M}$, mean flow irreversible heat flux $H_{M}$, the turbulence production related to the Reynolds stress $P_{R}$, turbulent heat flux $H_{R}$, and advection of TKE $A_{k}$. We remark that the terms $P_{R}, H_{R}$ and $A_{k}$ stand for the contribution of turbulence fluctuations to the irreversible process of entropy generation.

\section{Sub-Grid Scale Terms with LES Equations}

High-fidelity simulations, including DNS and LES, can be used for calibration of RANS solutions [13,14]. As DNS is usually too expensive for most turbomachinery flows, LES data seems to be more widely applied. In order to derive entropy generation terms for LES fields, the LES equations need to be investigated.

Defining a spatial filtering operation $\langle\cdot\rangle$, a Favre-filtered field is written as

$$
\widehat{\mathcal{C}}=\frac{\langle\rho \mathcal{C}\rangle}{\langle\rho\rangle} .
$$


Journal of Turbomachinery

Applying the Favre-filtering operation to Eqns. (4) and (5), the LES equations can be written as:

$$
\frac{\partial\left(\langle\rho\rangle \widehat{u}_{i}\right)}{\partial t}+\frac{\partial\left(\langle\rho\rangle \widehat{u}_{i} \widehat{u}_{j}+\langle p\rangle \delta_{i j}\right)}{\partial x_{j}}=\frac{\partial \widehat{\sigma}_{i j}}{\partial x_{j}}+\frac{\partial \tau_{i j}^{S G S}}{\partial x_{j}},
$$

and

$$
\begin{aligned}
\frac{\partial\left(\langle\rho\rangle \widehat{e}_{0}\right)}{\partial t}+\frac{\partial\left[\widehat{u}_{j}\left(\langle\rho\rangle \widehat{e}_{0}+\langle p\rangle\right)\right]}{\partial x_{j}}= & \frac{\partial\left(\widehat{\sigma}_{i j} \widehat{u}_{i}\right)}{\partial x_{j}}+\frac{\partial\left(\tau_{i j}^{S G S} \widehat{u}_{i}\right)}{\partial x_{j}} \\
& -\frac{\partial \widehat{q}_{j}}{\partial x_{j}}-\frac{\partial q_{j}^{S G S}}{\partial x_{j}}
\end{aligned}
$$

Here, two extra terms are introduced to account for the contribution of the sub-grid scale (SGS) fluctuations to the resolved flow, i. e. the SGS stress

$$
\tau_{i j}^{S G S}=-\langle\rho\rangle\left(\widehat{u_{i} u_{j}}-\widehat{u}_{i} \widehat{u}_{j}\right)
$$

and the SGS heat flux

$$
q_{j}^{S G S}=\langle\rho\rangle c_{p}\left(\widehat{T u_{j}}-\widehat{T} \widehat{u}_{j}\right)
$$

When we calculate entropy generation based on LES mean flow fields, these SGS terms also have a direct impact.

It is common to assume that taking the Favre-average of a filtered field is equivalent to taking the Favre-average of the original field

$$
\widetilde{\widehat{\mathcal{C}}}=\widetilde{\mathcal{C}} .
$$

Therefore, the entropy balance equation for Favre-averaged LES flow fields is similar to Eqn. (22), only with 
two extra SGS terms:

$$
\begin{aligned}
\frac{\partial\left(\bar{\rho} \widetilde{u_{i}} \widetilde{s}\right)}{\partial x_{i}} & +\frac{\partial}{\partial x_{i}}\left(\frac{\widetilde{q}_{i}}{\widetilde{T}}\right)=\frac{1}{\widetilde{T}} \sigma_{i j}(\widetilde{u}) \frac{\partial \widetilde{u}_{j}}{\partial x_{i}}+\frac{c_{p} \mu}{P r} \frac{1}{\widetilde{T}^{2}}\left(\frac{\partial \widetilde{T}}{\partial x_{i}}\right)^{2} \\
& +\frac{1}{\widetilde{T}} \tau_{i j}^{R} \frac{\partial \widetilde{u}_{j}}{\partial x_{i}}-\frac{1}{\widetilde{T}} \frac{\partial}{\partial x_{i}}\left(q_{i}^{R}\right)-\frac{1}{\widetilde{T}} \frac{\partial\left(\bar{\rho} \widetilde{u}_{i} k\right)}{\partial x_{i}} \\
& +\frac{1}{\widetilde{T}} \widetilde{\tau_{i j}^{S G S}} \frac{\partial \widetilde{u}_{j}}{\partial x_{i}}-\frac{1}{\widetilde{T}} \frac{\partial}{\partial x_{i}}\left(\widetilde{q_{i}^{S G S}}\right) .
\end{aligned}
$$

Thus the entropy generation for LES flow fields can be written as

$$
\begin{aligned}
\widetilde{G}_{s}^{L E S}= & \underbrace{\frac{1}{\widetilde{T}} \sigma_{i j}(\widetilde{u}) \frac{\partial \widetilde{u}_{j}}{\partial x_{i}}}_{V_{M}}+\underbrace{\frac{c_{p} \mu}{P r} \frac{1}{\widetilde{T}^{2}}\left(\frac{\partial \widetilde{T}}{\partial x_{i}}\right)^{2}}_{H_{M}} \\
& +\underbrace{\frac{1}{\widetilde{T}} \tau_{i j}^{R} \frac{\partial \widetilde{u}_{j}}{\partial x_{i}}}_{P_{R}}-\underbrace{\frac{1}{\widetilde{T}} \frac{\partial}{\partial x_{i}}\left(q_{i}^{R}\right)}_{H_{R}}-\underbrace{\frac{1}{\widetilde{T}} \frac{\partial\left(\bar{\rho} \widetilde{u}_{i} k\right)}{\partial x_{i}}}_{A_{k}} \\
& +\underbrace{\frac{1}{\widetilde{T}} \widetilde{\tau}_{i j}^{S G S} \frac{\partial \widetilde{u}_{j}}{\partial x_{i}}}_{V_{S}}-\underbrace{\frac{1}{\widetilde{T}} \frac{\partial}{\partial x_{i}}\left(\widetilde{q_{i}^{S G S}}\right)}_{H_{S}},
\end{aligned}
$$

where $V_{S}$ and $H_{S}$ represent the entropy generation related to the viscous dissipation caused by SGS stress and the SGS heat flux.

\section{NUMERICAL SIMULATIONS}

The entropy generation analysis is applied to a LS-89 HPT vane [15] operating at an isentropic exit Reynolds number of $R e=0.57 \times 10^{6}$ and an exit Mach number of $M a=0.9$, which is representative of a modern transonic HPT nozzle [10]. As the flow field shows complex flow physics including boundary layer transition, shock waves and vortex shedding in the blade wake, the selected case is challenging for RANS calculations. In the present study, the available high-resolution LES data [16] is first post-processed, and then RANS calculations are performed and analysed. The error source for the RANS predictions are quantitatively investigated by comparing to the LES results. 


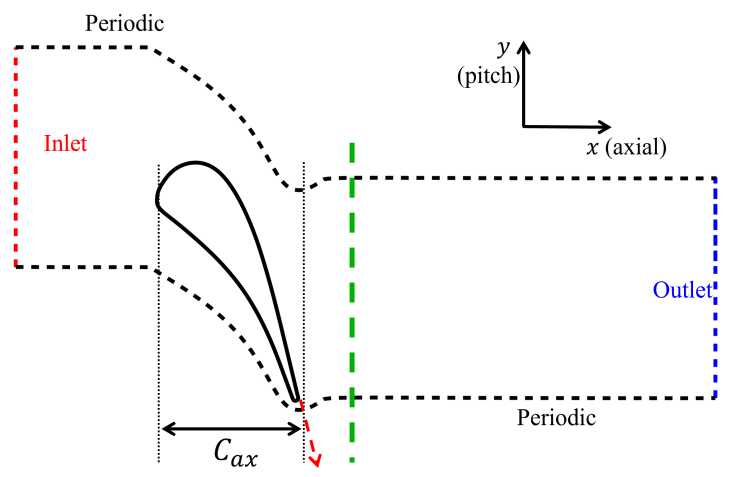

Fig. 1. Sketch of the LS89 HPT blade geometry and domain boundaries.

\section{LES Data}

The LES was performed using the in-house compressible Navier-Stokes solver HiPSTAR, with the standard WALE model [17] applied as the SGS model. The numerical solver has been extensively verified for turbomachinery flows [18]. The details of the data was presented in a previous study [16], and only a brief summary of the key features of the case is presented here.

The geometry of an axial-pitch plane cut of the HPT computational domain is shown in Fig. 1. The axial chord $C_{a x}$ is used as the reference length scale, and the blade surface is set as an iso-thermal wall. Periodic conditions are applied at the boundaries in the pitchwise direction $(y)$, and a zonal characteristic boundary-condition [19] is applied to attenuate acoustic reflections at the axial $(x)$ outlet boundary. At the inlet boundary, Riemann invariant boundary conditions have been used, along with additional turbulence fluctuations generated by the digital filter method [20]. The integral length scale of the turbulence fluctuations is $20 \% C_{a x}$ and the intensity $T I$ is $6 \% u_{\infty}$. The turbulence intensity is defined as $T I=\frac{\sqrt{2 / 3 k}}{u_{\infty}}$, where the reference velocity $u_{\infty}$ is set as the inlet mean velocity. The HPT is statistically two dimensional as periodic conditions are applied at the boundaries in the spanwise direction. The spanwise domain size is set as $L_{z}=80 \% C_{a x}$, which allows for the spanwise correlation of the large turbulence length scale. The wall normal spacing of the LES mesh is below $y^{+}=2$, and the spacing in the spanwise and axial directions are around 20 and 50 wall units, respectively. This extraordinary high-resolution guarantees the accuracy of the LES data, which has been compared against [16] the DNS results of Wheeler et al. [10].

\section{RANS Setup}

RANS calculations of the HPT case have been performed using the finite volume solver TRAF [21]. The two dimensional Reynolds-averaged Navier-Stokes equations are solved on multi-block structured grids. 
Table 1. RANS grid convergence parameters.

\begin{tabular}{clll}
\hline Grids & O-Grid & H-Grid & Total Points \\
\hline Coarse & $565 \times 57$ & $81 \times 101$ & 40,386 \\
Mid & $705 \times 65$ & $101 \times 161$ & 62,086 \\
Fine & $1081 \times 113$ & $161 \times 320$ & 173,673 \\
\hline
\end{tabular}

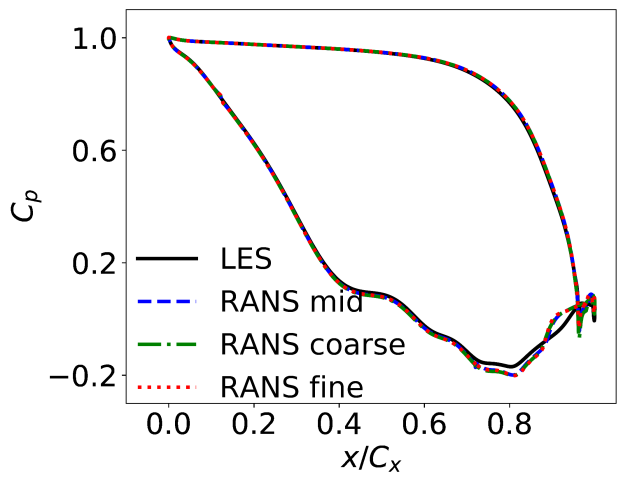

Fig. 2. Pressure coefficient distribution across the blade.

The convective fluxes are discretized by a $2^{\text {nd }}$ order TVD-MUSCL strategy build on Roe's upwind scheme, and the viscous fluxes are solved by a central difference scheme. For turbulence closures, the $k-\omega$ SST with $\gamma-R e_{\theta}$ transitional correlation [22] is applied. The computational domain was discretized using an $\mathrm{O}-\mathrm{H}$ grid with $705 \times 65$, and $101 \times 161$ mesh points, respectively. The mesh was selected on the basis of a grid-refinement analysis. The dimensions for the three different grids to establish grid independence are listed in Tab. 1.

The RANS results are compared to the LES data. The pressure coefficients $C_{p}$, obtained from these simulations, are plotted versus the fraction of axial chord in Fig. 2. Starting from the leading edge, the suction-side pressure drops quickly, while the decrease of $C_{p}$ on the pressure-side initiates near the trailing edge. All the RANS cases capture the pressure decrease on both sides, and a good agreement is shown between the RANS and LES results. Moreover, the RANS results show a slight deviation from the LES data near the suction-side trailing edge, which is presumably due to the boundary layer transition not being captured correctly by the RANS.

The transition on the blade can be presented by the wall friction coefficients shown in Fig. 3. For the pressure-side and the early suction-side boundary layer, the RANS calculations provide an accurate 


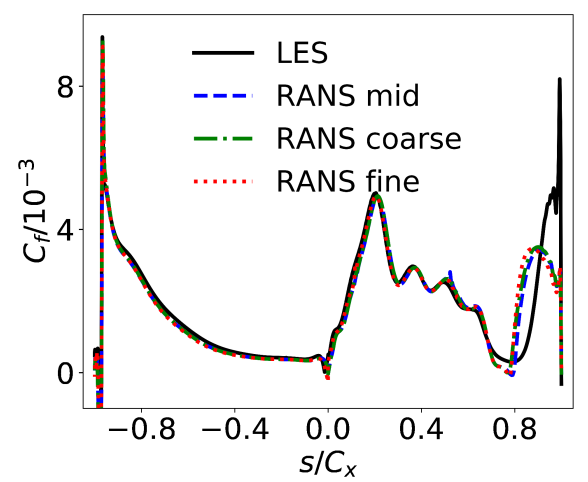

Fig. 3. Wall friction distribution across the blade.

prediction of the wall friction. Moreover, the onset of the suction-side transition is captured by the $\gamma-\operatorname{Re}_{\theta}$ model. However, the region in close proximity to the trailing edge is not predicted well. Despite the deviation near the trailing edge, the grid convergence is demonstrated with the RANS meshes.

Furthermore, Fig. 4 shows the kinetic wake loss profiles at $20 \% C_{a x}$ downstream of the blade trailing edge as indicated by the green dashed line in Fig. 1. The kinetic wake loss is defined as

$$
\omega\left(y^{*}\right)=\frac{p_{t, i}-p_{t, o}\left(y^{*}\right)}{p_{t, i}-p_{o}},
$$

with the normalized pitchwise coordinate $y^{*}=\left(y-y_{\min }\right) /\left(y_{\max }-y_{\min }\right)$. Here, $p_{t, i}, p_{o}$, and $p_{t, o}$ denote the inlet total pressure, outlet static pressure, and outlet total pressure, respectively. We can see that the RANS calculations significantly over-predict the wake peak and under-predict the wake width. The failure of capturing the wake mixing in turbine flows is known as a common weakness for traditional RANS models [14], and its impact will now be investigated based on the entropy analysis.

\section{ENTROPY GENERATION ANALYSIS IN HPT VANE Quantification of Entropy Generation Terms}

The entropy analysis shown in the present study is applied to both LES and RANS flow fields. The LES data have been averaged in time and in the spanwise direction, and the entropy generation terms shown in Eqn. (31) are calculated based on the averaged LES statistics. On the other hand, the turbulence-related terms from RANS are calculated based on the turbulence models. Therefore, the method provides a way 


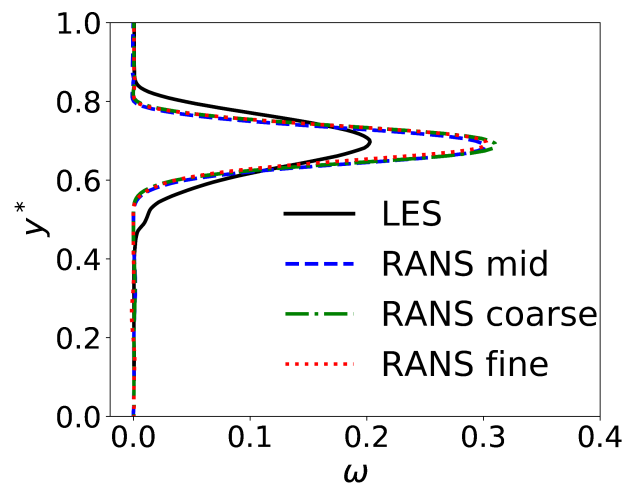

Fig. 4. Wake loss profiles at $20 \%$ downstream of the trailing edge $\left(x=1.2 C_{a x}\right)$.

Table 2. Quantification of entropy generation terms in LES and RANS.

\begin{tabular}{crrr}
\hline Terms $\left(J K^{-1} s^{-1}\right)$ & LES int. & RANS int. & Error \\
\hline$V_{M}$ & 0.0367 & 0.0344 & 0.0023 \\
$H_{M}$ & 0.0116 & 0.0096 & 0.0020 \\
$P_{R}$ & 0.0598 & 0.0398 & 0.0200 \\
$H_{R}$ & -0.0003 & 0.0015 & -0.0018 \\
$A_{k}$ & -0.0199 & -0.0070 & -0.0129 \\
$V_{S}$ & 0.0026 & N.A. & N.A. \\
$H_{S}$ & 0.0008 & N.A. & N.A. \\
\hline$G_{s}$ & 0.0913 & 0.0783 & 0.0130 \\
$-\dot{m} R \ln \left(P_{t, o} / P_{t, i}\right)$ & 0.0926 & 0.0797 & 0.0129 \\
\hline
\end{tabular}

to quantify the modelling error from RANS by comparing to LES.

In order to quantify the loss increase caused by different terms, volume integrals of the entropy generation equations Eqn.(23) and Eqn.(31) are performed. The control volume can be selected as the vane between the inlet and outlet planes as shown in Fig. 1. This volume integral is applied to both the LES data and the RANS results, and the total entropy generation by different terms are listed in Tab. 2.

These terms can be divided into three groups. The first group represents the mean flow effects including the mean viscous dissipation $V_{M}$ and the mean heat flux $H_{M}$. The second group includes the entropy generation induced by turbulence fluctuations, containing the turbulence production $P_{R}$, the turbulent heat flux $H_{R}$, and the advection of TKE $A_{k}$. And the last group consists of the SGS stress terms $V_{S}$ and the SGS 
heat flux term $H_{S}$, which only apply for the LES data.

Among these groups, the contributions from the mean flow and the turbulence fluctuations are on roughly the same level, and the viscous effects in each group have much higher amplitudes than the heat flux terms, as $V_{M}>H_{M}$ and $P_{R}>H_{R}$. On the other hand, the contribution of the SGS terms is relatively small, as $\left(V_{S}+H_{S}\right) / G_{s} \approx 3.7 \%$. We note that the amplitude of the entropy generation caused by the SGS terms mainly depends on the grid resolution and overall turbulence intensity in the flow field. The small SGS contribution implies the present LES flow is highly-resolved. Nevertheless, to fully avoid any uncertainty, a DNS might be desirable.

As the averaged flow field is time-invariant, the total entropy generation $G_{s}$ integrated over the whole HPT vane should equal to the entropy flux with the environment. The volume integrals of the left-hand side entropy flux terms from Eqn. (22) or Eqn. (30) can be simplified as the mass-flux term

$$
\begin{aligned}
\int_{V} \frac{\partial\left(\bar{\rho} \widetilde{u_{i}} \widetilde{s}\right)}{\partial x_{i}} d V & =\int_{A} \bar{\rho} \widetilde{u_{i}} \widetilde{s} d A \\
& =\dot{m}\left(\widetilde{s}_{\text {out }}-\widetilde{s}_{\text {in }}\right),
\end{aligned}
$$

and the heat transfer term

$$
\int_{V} \frac{\partial}{\partial x_{i}}\left(\frac{\widetilde{q}_{i}}{\widetilde{T}}\right) d V=\int_{A_{b}} \frac{\widetilde{q}_{i}}{\widetilde{T}} d A
$$

As we can see, the mass-flux induced entropy transport is represented by the specific entropy difference between the outlet and inlet plane multiplied by the mass flow rate $\dot{m}$, whereas the entropy transport caused by heat transfer is dominated by the heat flux on the blade surface $A_{b}$. These two terms depict the overall entropy transfer between the HPT vane with the environment, $i$. e. the upstream and downstream stages and the turbine blade.

From basic thermodynamics, the specific entropy of the averaged single phase fluid can be written as

$$
\widetilde{s}-\widetilde{s}_{r e f}=c_{p} \ln \left(T_{t} / T_{t, r e f}\right)-R \ln \left(P_{t} / P_{t, r e f}\right),
$$


where $R$ is gas constant. Conventionally, the kinetic loss in the turbine vane can be represented by ignoring the blade heat flux and the temperature term [3], as

$$
-\dot{m} R \ln \left(P_{t, o} / P_{t, i}\right) \approx \int_{V} \widetilde{G}_{s} d V
$$

On the left-hand side, the $-\dot{m} R \ln \left(P_{t, o} / P_{t, i}\right)$ term is the entropy variation related to the total pressure difference between the outlet and inlet plane, which is calculated based on the LES and RANS flow field and presented in Tab. 2. This entropy difference is closely related to the total entropy generated in the vane as indicated by the right-hand side term $\int_{V} \widetilde{G}_{s} d V$. We can see from Tab. 2 that the balance in Eqn. (36) is approximately satisfied, which indicates that the entropy generation terms presented in this study are quantitatively linked to the kinetic loss in the vane.

Furthermore, we can compare the terms from LES and RANS calculations in Tab. 2. It is shown that the major difference is caused by the turbulence production term $P_{R}$. The turbulence production represents the energy extracted from the mean flow and passed on to turbulent fluctuations. Negative production is possible in LES when the TKE inversely cascades to mean kinetic energy, but it is not allowed by the RANS approach. Most of the TKE will not be available for mechanical work but will gradually dissipate away. Therefore, the TKE may be considered as one kind of 'pseudo internal energy' in the present entropy calculations, and the advection term $A_{k}$ represents the redistribution of TKE, which also induces entropy variation. It is noted that the contribution of $A_{k}$ for the whole vane is negative as presented in Tab. 2 .

\section{Spatial Distribution of Entropy Generation}

The spatial distribution of the entropy generation in the turbine vane is investigated to further understand the physical mechanisms. The LES spatial distribution of the entropy generation caused by the mean flow viscous dissipation $V_{M}$ is first presented in Fig. 5. We can see that the mean flow viscous dissipation is mainly in the blade boundary layer, and the amplitude in the rest of the vane is negligible.

In addition, a contour of the turbulence production term $P_{R}$ from LES is shown in Fig. 6 . The entropy generated by turbulence production concentrates in two regions, including the late suction-side boundary layer, related to the laminar-turbulent transition, and the wake region, which corresponds to the vortex shedding downstream the trailing edge. For conciseness, the contours for the other terms in Eqn. (31) are not shown here. However, we can infer from Fig. 5 and Fig. 6 that the mean flow effects mainly manifest 


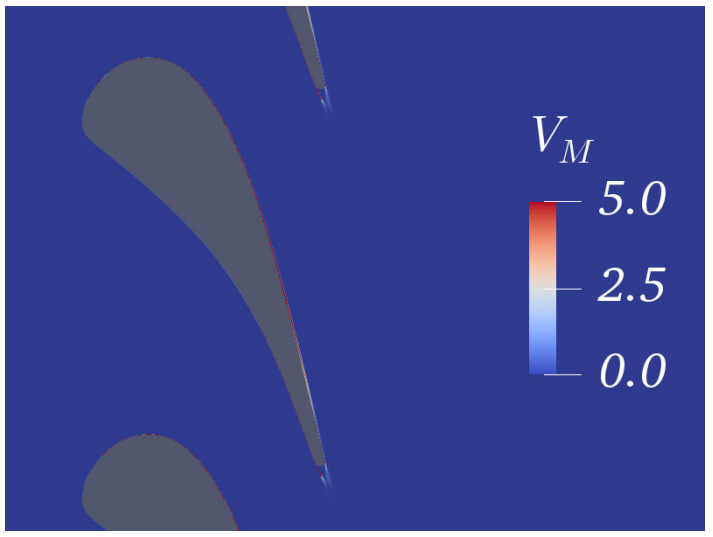

Fig. 5. Distribution of the entropy generation caused by mean viscous dissipation $V_{M}$.

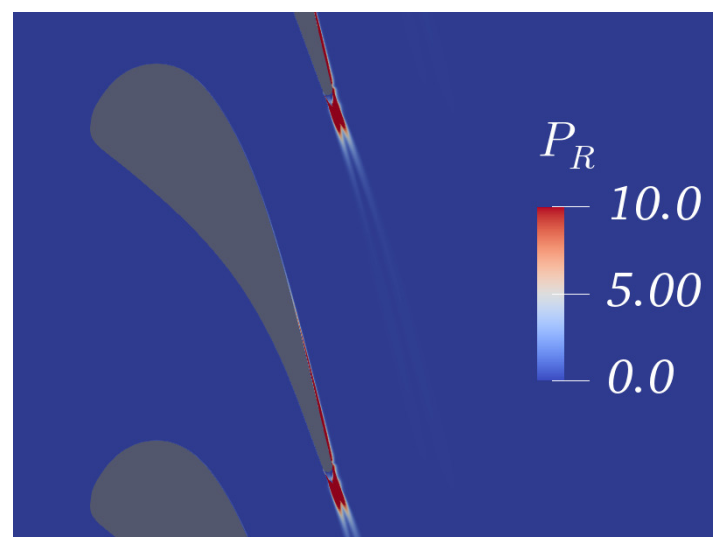

Fig. 6. Distribution of the production-induced entropy generation $P_{R}$.

themselves in the blade boundary layer, while the turbulence effects are caused by the suction-side turbulent boundary layer and the strong vortex shedding in the wake.

The rise in entropy generation by the mean flow effects is quantified in Fig. 7 by integrating over the volume between the inlet plane to a downstream axial plane at $x$. As the mean flow entropy generation occurs mainly in the blade boundary layer, the increase of $V_{M}$ and $H_{M}$ are distributed between the blade leading edge $(x=0)$ and trailing edge $(x=1)$ as indicated by the yellow dashed lines in Fig. 7 . The rate of increase of $H_{M}$ at the leading edge is larger, indicating a stronger temperature gradient in the boundary layer. On the other hand, the rate of increase of the viscous dissipation in the boundary layer is increasing along the blade surface, because the shear is getting stronger as the flow accelerates and the Reynolds number increases. It is noted that the entropy generation terms from these mean flow effects remain approximately invariant downstream the trailing edge, and the mean viscous dissipation has a much larger 


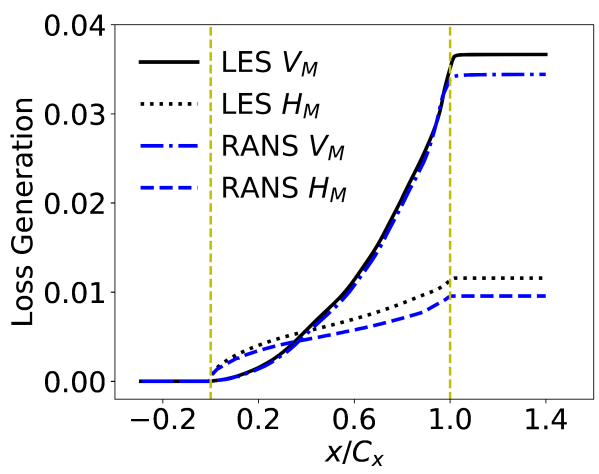

Fig. 7. Integrals of the entropy generation caused by mean flow.

amplitude compared to the heat flux effect.

In addition, LES and RANS results in Fig. 7 can be directly compared. It is shown that the mean viscous dissipation $V_{M}$ from RANS agrees well with the LES result until the blade trailing edge, implying that the velocity boundary layer is accurately predicted by the RANS calculation. A relatively small deviation exists after the blade trailing edge, which is presumably because the RANS-predicted shear layer in the wake close to the trailing edge is not as strong as the shear layer from LES [10]. Moreover, the RANS-predicted $H_{M}$ deviates from the LES result, while the curves have a similar trend. The difference implies that the RANS thermodynamic boundary layer around the blade surface is not as accurate as the velocity field, suggesting a model form error of the heat flux model [23].

Furthermore, the entropy generation terms related to turbulence effects are shown in Fig. 8. The turbulence-production related entropy generation is dominating for both the LES and RANS results. From the blade leading edge, the production caused entropy generation barely increases until the suction-side boundary layer transition. The RANS calculation provides a satisfactory prediction around the blade surface, including the evident increase of entropy generation in the turbulent boundary layer. However, the major error between the LES and RANS seems to be due to the turbulence production downstream of the blade trailing edge. Compared to RANS, the LES shows a more significant turbulence production just downstream of the trailing edge. Nevertheless, the production rate further downstream is negligible for both cases.

The deviation between the turbulence production downstream of the blade trailing edge will result in different amplitudes of TKE in the wake. The TKE distributions are plotted along the wake center line indicated by the red dashed arrow in Fig. 1. It is presented in Fig. 9 that the RANS calculation has a much lower TKE peak compared to the LES result, which is mainly due to the smaller amplitude of turbulence 


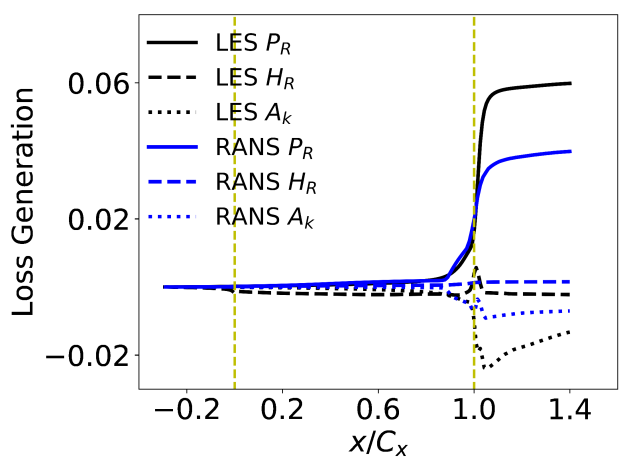

Fig. 8. Integrals of the entropy generation caused by turbulence effects.

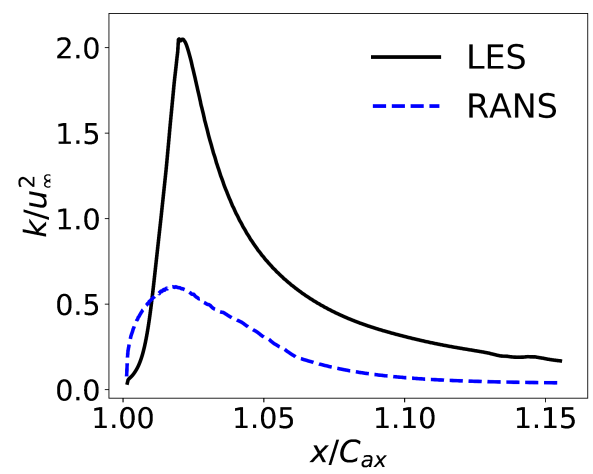

Fig. 9. The TKE distribution along the wake center line.

production. Furthermore, the distribution of TKE has an effect on the advection term $A_{k}$. As shown in Fig. 8, the TKE advection $A_{k}$ from RANS calculation significantly deviates from the LES result, which is because of the difference of the TKE distributions near the blade trailing edge. However, the error of $A_{k}$ gradually decreases further downstream, so that the contribution for the total entropy variation is not as significant as $P_{R}$.

The turbulence heat flux term indicates the heat transfer induced by turbulent fluctuations. Although the contribution compared to the turbulence production term is negligible, the difference between the RANS and LES results implies the limitation of the turbulent heat flux modelling [23], particularly in the near trailing edge region with strong vortex shedding. 


\section{CONCLUSION}

In the present study, we present the derivation of the entropy balance equations for Favre-averaged flow fields. The equations are developed from basic flow-governing equations and thermodynamic laws, so that the framework can be applied to a wide range of data sets including RANS and high-fidelity simulations. The entropy generation, which is closely related to the loss of efficiency of the system, is decomposed into different terms such as mean viscous dissipation, turbulence production, mean and turbulent heat flux, etc.

This decomposition framework is then applied to available high-resolution LES and RANS results of the transonic HPT case. A quantitative analysis of these terms shows that the contributions from the mean flow terms and the turbulence terms are on a similar level, while the SGS terms from the LES data only have a weak effect in this HPT case. Adding all these terms up, the total entropy generated in the whole HPT vane provides a good approximation for the kinetic loss.

Furthermore, the spatial distributions of the entropy generation terms have been investigated, and differences between the LES data and the RANS calculations have been quantitatively analysed. It is shown that the entropy generated by the mean flow effects concentrate in the blade boundary layer, while the turbulence fluctuations induce entropy increase in the suction-side turbulent boundary layer and the wake region with vortex shedding. In particular, the turbulence production term, which represents the energy extracted from the mean flow to turbulent fluctuations, plays an important role in entropy generation. However, the difference between RANS and LES results for the turbulence production term is not negligible, especially in the wake region near the blade trailing edge. This implies that the error of RANS prediction is mainly caused by the inaccurate turbulence production modelled in the near wake region.

\section{ACKNOWLEDGEMENTS}

This research used resources of the Oak Ridge Leadership Computing Facility, which is a DOE Office of Science User Facility supported under Contract DE-AC05-00OR22725. This work was also supported by the resources provided by the Pawsey Supercomputing Centre with funding from the Australian Government and the Government of Western Australia.

\section{NOMENCLATURE}

\section{General Symbols}

$C_{a x} \quad$ Blade axial chord

$C_{p}$ Pressure coefficient 
$C_{f} \quad$ Wall friction coefficient

$c_{p} \quad$ Specific heat at constant pressure

e Internal energy

$e_{0} \quad$ Total energy

$k$ Turbulent kinetic energy

Ma Mach number

$\dot{m}$ Mass flow rate

$\operatorname{Pr}$ Prandtl number

$p$ Pressure

$q_{i} \quad$ Heat flux vector

$R$ Gas constant

Re Reynolds number

$T$ Temperature

TI Turbulence intensity

$x \quad$ Axial coordinate, normalized by axial chord

y Pitchwise coordinate, normalized by axial chord

\section{Symbols Related to Entropy}

$A_{k} \quad$ Entropy generation by TKE advection

$F_{i} \quad$ Entropy flux vector

$G_{s}$ Entropy generation rate

$H_{M} \quad$ Entropy generation by mean flow heat flux

$H_{R} \quad$ Entropy generation by turbulent heat flux

$H_{S} \quad$ Entropy generation by SGS heat flux

$P_{R} \quad$ Entropy generation by turbulence production

$S$ System total entropy

$s \quad$ Specific entropy

$V_{M} \quad$ Entropy generation by mean viscous dissipation

$V_{S} \quad$ Entropy generation by SGS stress dissipation 


\section{Greek Letters}

$\mu$ Dynamic viscosity

$\mu_{t} \quad$ Eddy viscosity

$\rho$ Density

$\omega$ Kinetic wake loss

$\sigma_{i j}$ Viscous stress tensor

$\tau_{i j}^{R} \quad$ Reynolds stress tensor

$\tau_{i j}^{S G S} \quad$ Sub-grid scale stress tensor

\section{Accents}

- Time-average

Favre-average

. Favre-filter

\section{Acronyms}

CFD Computational fluid dynamics

DNS Direct numerical simulation

HPT How-pressure turbine

LES Large-eddy simulation

LPT Low-pressure turbine

RANS Reynolds averaged NavierStokes

SGS Sub-grid scale

TKE Turbulent kinetic energy

\section{REFERENCES}

[1] Bejan, A., 1978. "General criterion for rating heat-exchanger performance". Int. J. Heat Mass Tran., 21(5), pp. 655-658.

[2] Bejan, A., 1980. "Second law analysis in heat transfer". Energy, 5(8-9), pp. 720-732.

[3] Denton, J. D., 1993. "Loss Mechanisms in Turbomachines". ASME J. Turbomach., 115(4), p. 621.

[4] Denton, J. D., 2010. "Some limitations of turbomachinery CFD". In ASME Turbo Expo 2010: Power for Land, Sea, and Air, pp. 735-745. 
[5] Sciubba, E., 1997. "Calculating Entropy with CFD". ASME Mech. Eng., 119(10), p. 86.

[6] Naterer, G. F., and Camberos, J. A., 2003. "Entropy and the Second Law Fluid Flow and Heat Transfer Simulation". J. Thermophys. Heat Tr., 17(3), pp. 360-371.

[7] Moore, J., and Moore, J. G., 1983. "Entropy Production Rates from Viscous Flow Calculations: Part I-A Turbulent Boundary Layer Flow". ASME Paper No. 83-GT-70.

[8] Camberos, J. A., 2001. "On the Construction of Entropy Balance Equations for Arbitrary Thermophysical Processes". In Proc. 39th AIAA Aerosp. Sci. Meet., no. 2001, p. 0815.

[9] Adeyinka, O. B., and Naterer, G. F., 2004. "Modeling of Entropy Production in Turbulent Flows". J. Fluids Eng., 126(6), p. 893.

[10] Wheeler, A. P. S., Sandberg, R. D.and Sandham, N. D., Pichler, R., and Michelassi, V., 2016. "Direct Numerical Simulations of a High-Pressure Turbine Vane". ASME J. Turbomach., 138(7), p. 071003.

[11] Lengani, D., Simoni, D., Pichler, R., Sandberg, R. D., Michelassi, V., and Bertini, F., 2018. "Identification and Quantification of Losses in a LPT Cascade by POD Applied to LES Data". Int. J. Heat Fluid Flow, 70, pp. 28-40.

[12] Adumitroaie, V., Ristorcelli, J. R., and Taulbee, D. B., 1999. "Progress in Favré-Reynolds Stress Closures for Compressible Flows". Phys. Fluids, 11(9), pp. 2696-2719.

[13] Poroseva, S. V., Colmenares, J. D., and Murman, S. M., 2016. "On the Accuracy of RANS Simulations with DNS Data". Phys. Fluids, 28(11), p. 115102.

[14] Pichler, R., Sandberg, R. D., Michelassi, V., and Bhaskaran, R., 2016. "Investigation of the Accuracy of RANS Models to Predict the Flow Through a Low-Pressure Turbine". ASME J. Turbomach., 138(12), p. 121009.

[15] Arts, T., Lambertderouvroit, M., and Rutherford, A. W., 1990. Aero-thermal Investigation of a Highly Loaded Transonic Linear Turbine Guide Vane Cascade. Tech. rep., von Karman Institute for Fluids Dynamics, Brussels, Belgium.

[16] Pichler, R., Sandberg, R. D., Laskowski, G., and Michelassi, V., 2017. "High-Fidelity Simulations of a Linear HPT Vane Cascade Subject to Varying Inlet Turbulence". ASME paper No. GT2017-63079.

[17] Nicoud, F., and Ducros, F., 1999. "Subgrid-scale stress modelling based on the square of the velocity gradient tensor". Flow Turbul. Combust., 62(3), pp. 183-200.

[18] Sandberg, R. D., Michelassi, V., Pichler, R., Chen, L., and Johnstone, R., 2015. "Compressible Direct Numerical Simulation of Low-Pressure Turbines-Part I: Methodology". ASME J. Turbomach., 137(5), p. 051011. 
[19] Sandberg, R. D., and Sandham, N. D., 2006. "Nonreflecting Zonal Characteristic Boundary Condition for Direct Numerical Simulation of Aerodynamic Sound". AIAA J., 44(2), pp. 402-405.

[20] Klein, M., Sadiki, A., and Janicka, J., 2003. "A digital filter based generation of inflow data for spatially developing direct numerical or large eddy simulations". J. Comput. Phys., 186(2), pp. 652-665.

[21] Arnone, A., and Pacciani, R.and Sestini, A., 1995. "Multigrid Computations of Unsteady Rotor-Stator Interaction Using the Navier-Stokes Equations". J. Fluids Eng., 117(4), pp. 647-652.

[22] Langtry, R. B., and Menter, F. R., 2009. "Correlation-based Transition Modeling for Unstructured Parallelized Computational Fluid Dynamics Codes". AIAA J., 47(12), pp. 2894-2906.

[23] Sandberg, R. D., Tan, R., Weatheritt, J., Ooi, A., Haghiri, A., Michelassi, V., and Laskowski, G., 2018. "Applying Machine Learnt Explicit Algebraic Stress and Scalar Flux Models to a fundamental Trailing Edge Slot”. ASME J. Turbomach., 140(10), p. 101008. 


\section{LIST OF FIGURES}

1 Sketch of the LS89 HPT blade geometry and domain boundaries. . . . . . . . . . 11

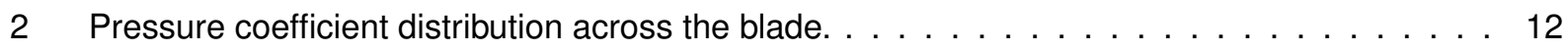

3 Wall friction distribution across the blade. $\ldots \ldots \ldots \ldots \ldots$

4 Wake loss profiles at $20 \%$ downstream of the trailing edge $\left(x=1.2 C_{a x}\right) \ldots \ldots \ldots$

5 Distribution of the entropy generation caused by mean viscous dissipation $V_{M} \ldots \ldots \ldots$

6 Distribution of the production-induced entropy generation $P_{R} \ldots \ldots \ldots \ldots$

7 Integrals of the entropy generation caused by mean flow. $\ldots \ldots \ldots \ldots$

8 Integrals of the entropy generation caused by turbulence effects. $\ldots \ldots \ldots \ldots$

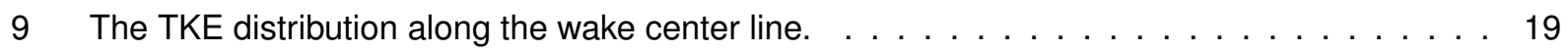




\section{LIST OF TABLES}

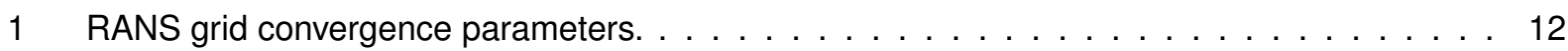

2 Quantification of entropy generation terms in LES and RANS . . . . . . . . . . . . . . 14 


\section{University Library}

\section{- M M I N E R VA A gateway to Melbourne's research publications}

Minerva Access is the Institutional Repository of The University of Melbourne

Author/s:

Zhao, Y;Sandberg, RD

Title:

Using a New Entropy Loss Analysis to Assess the Accuracy of RANS Predictions of an HighPressure Turbine Vane

Date:

2020-08-01

\section{Citation:}

Zhao, Y. \& Sandberg, R. D. (2020). Using a New Entropy Loss Analysis to Assess the Accuracy of RANS Predictions of an High-Pressure Turbine Vane. Journal of Turbomachinery, 142 (8), https://doi.org/10.1115/1.4046531.

Persistent Link:

http://hdl.handle.net/11343/241882 